Philip C. Vergeiner, Elisabeth Buchner, Eva Fuchs, Stephan Elspaß

\title{
Weil STANDARD verständlich ist und DIALEKT authentisch macht
}

\author{
Varietätenkonzeptionen im sekundären und tertiären \\ Bildungsbereich in Österreich
}

Zusammenfassung: Der vorliegende Beitrag zielt darauf ab, Varietätenkonzeptionen im sekundären und tertiären Bildungsbereich in Österreich zu analysieren. Dabei wird gefragt, (a) was Sprecher/innen an Schule und Universität unter Varietäten wie DIALEKT und STANDARD verstehen, (b) wie sie sie jeweils bewerten und (c) welche normativen Erwartungen sie zu ihrer Verwendung in Bildungskontexten haben. Um diese Fragen zu beantworten, wurden halbstrukurierte Interviews einer inhaltsanalytischen Untersuchung zugeführt. Die Ergebnisse zeigen, dass es sowohl an Schule als auch Universität zum Teil ähnliche Varietätenkonzepte gibt. Zugleich werden jedoch auch Ambiguitäten und Widersprüche zwischen beiden Bereichen ebenso wie innerhalb dieser offengelegt. Dies spiegelt die komplexe DIAGLOSSISCHE, z. T. auch DIGLOSSISCHE Situation in Österreich, wo DIALEKT und STANDARD im Alltag gleichermaßen präsent sind und einzelne Varietäten regionen-, situations- und wohl auch individuenspezifisch unterschiedlich bewertet und verwendet werden.

Abstract: The aim of this paper is to analyse concepts of language varieties in secondary and tertiary education in Austria. The questions put forward are (a) what speakers' notions of varieties such as dialect and standard are at schools and universities, (b) how they evaluate them and (c) what normative expectations they have for their use in educational contexts. To answer these questions, semi-structured interviews were subjected to a content analysis. The results

\footnotetext{
Vergeiner, Philip C.: Universität Salzburg, FB Germanistik, Erzabt-Klotz.Straße 1, A-5020 Salzburg, philip.vergeiner@sbg.ac.at

Buchner, Elisabeth: Universität Salzburg, FB Germanistik, Erzabt-Klotz.Straße 1, A-5020 Salzburg, elisabeth.buchner@sbg.ac.at

Fuchs, Eva: Pädagogische Hochschule Salzburg „Stefan Zweig“, Akademiestraße 23-25, A5020 Salzburg, eva.fuchs@phsalzburg.at

Elspaß, Stephan: Universität Salzburg, FB Germanistik, Erzabt-Klotz.Straße 1, A-5020

Salzburg, stephan.elspass@sbg.ac.at
} 
show that there are some similar variety concepts at school and university. At the same time, however, ambiguities and contradictions between the two areas as well as within them are disclosed. This reflects the complex diaglossic and partly also diglossic situation in Austria, where dialect and standard are equally present in everyday life and individual varieties are evaluated and used differently depending on the region, situation and probably also on an individual basis.

Schlüsselwörter: Standard, Dialekt, Varietätenkonzeptionen, Sprachwahrnehmungen, Spracheinstellungen, Normen

\section{Problemaufriss}

Bildungsinstitutionen galten lange als die Domäne der STANDARDSPRACHE - dies wurde für den tertiären Bildungsbereich als ebenso selbstverständlich angenommen (vgl. zusammenfassend Dannerer 2018: 169, 174-175) wie für den sekundären (vgl. bspw. Mattheier 1991). Der Gebrauch von NonSTANDARDVarietäten wurde daher auch - ganz im Sinne der Sprachbarrieren-Hypothese (vgl. Ammon 1972) - als etwas Defizitäres und von allen Beteiligten Unerwünschtes dargestellt. Mehrere Untersuchungen konnten seither jedoch nachweisen, dass in Bildungseinrichtungen NONSTANDARD-Gebrauch sehr wohl häufig vorkommt - zumindest im Süden des deutschsprachigen Raums (vgl. bspw. Knöbl 2012; Steiner 2008). Anzunehmen ist, dass dies der Fall ist, weil die Sprecher/innen an diesen Institutionen keine so negative Sicht auf den NONSTANDARD haben, wie ihnen früher bisweilen unterstellt wurde. Festzustellen ist jedoch, dass sowohl die tatsächliche Varietätenverwendung als auch die Konzepte von STANDARD und NONSTANDARD bei den Sprecher/inne/n im Bildungsbereich bis heute nur ausschnittweise untersucht wurden (vgl. für die Varietätenkonzepte von bundesdeutschen Deutschlehrer/inne/n bspw. Hochholzer 2004). Gerade in Österreich liegen zwar zur allgemeinen Sicht auf Varietäten viele Befunde vor (vgl. z. B. Bellamy 2012; Moosmüller 1991; Soukup 2009), in Hinblick auf Schule und Universität ist darüber aber nur wenig bekannt. ${ }^{1}$ Hier besteht ein klares Forschungsdesiderat.

1 Erste Ergebnisse zur Schule wurden im Rahmen des Projekts Österreichisches Deutsch als Unterrichts- und Bildungssprache erbracht, das sich allerdings stark auf den STANDARDNAHEN Bereich und verschiedene STANDARD-Varietäten des Deutschen fokussierte; vgl. bspw. de Cillia 
Ziel des vorliegenden Beitrages ist es, sich dieses Desiderats anzunehmen. Gefragt werden soll, welche Varietätenkonzeptionen verschiedene Sprecher/innen in sekundären und tertiären Bildungsinstitutionen zu STANDARDVarietäten einerseits und NONSTANDARD-Varietäten andererseits haben. Dabei sollen auch Gemeinsamkeiten und Unterschiede zwischen den Institutionen herausgearbeitet werden. Dazu werden Interviewdaten inhaltsanalytisch untersucht, die in zwei Projekten entstanden sind: zum einen im Projekt VAMUS (= Verknüpfte Analyse von Mehrsprachigkeiten am Beispiel der Universität Salzburg), ${ }^{2}$ zum anderen im Teilprojekt PP10 Wahrnehmung von und Einstellung zu Varietäten und Sprachen an österreichischen Schulen des Spezialforschungsbereichs Deutsch in Österreich (DiÖ). ${ }^{3}$

In der Folge wird zunächst geklärt, was wir im vorliegenden Aufsatz unter Laienkonzeptionen verstehen (Abschnitt 2). In Abschnitt 3 werden wir auf das Datenmaterial der Untersuchung eingehen, bevor wir in Abschnitt 4 die Untersuchungsergebnisse präsentieren. Abschließend erfolgt eine Diskussion mit Fazit (Abschnitt 5).

\section{Konzeptionelle Grundlage}

Die Sichtweise linguistischer Laien auf Sprachen und Varietäten wird bereits seit längerem ausgiebig beforscht. Dies geschieht unter Bezug auf verschiedene Konzepte - zu nennen sind etwa EInstellungen, IdEOlogien, PERZEPTIONEN oder NORMEN. Nicht immer ist klar, ob und inwiefern diese Begriffe klar abgrenzbar sind. In der Folge sollen die einzelnen Begriffe kurz diskutiert werden, um letztlich für einen holistischen Blickwinkel auf diese Phänomene in Form von ,Varietätenkonzeptionen' $z u$ plädieren.

Eines der ersten Paradigmen, welches die Laiensicht auf Sprache systematisch untersuchte, war die Einstellungsforschung. Ausgehend von sozialpsychologischen Zugängen werden EINSTELLUNGEN dabei bis heute häufig mit der klassischen Definition von Allport (1935: 810) als sozial erlernte Dispositionen

(2018). Zu Varietätenverhältnissen an der Universität kann vor allem auf Publikationen im Kontext des VAMUS-Projekts verwiesen werden, bspw. Dannerer (2018) oder Vergeiner (2021).

2 Das von Monika Dannerer und Peter Mauser geleitete Projekt wurde vom Jubiläumsfonds der Österreichischen Nationalbank finanziert (ÖNB-Projektnummer 15.827).

3 Das von Stephan Elspaß geleitete Projekt - Fonds zur Förderung der wissenschaftlichen Forschung (FWF), Projektnummer F 6010 G23 - wird als Teil des SFBs F 60 „Deutsch in Österreich" vom FWF gefördert. 
verstanden, die beeinflussen, wie auf gewisse Phänomene (z. B. Sprachen oder Varietäten) reagiert wird - dies im Denken (kognitiv), Werten (affektiv) und Handeln (konativ) (vgl. z. B. Ajzen 1989; aus linguistischer Sicht Lasagabaster 2004). Untersucht wurden Spracheinstellungen häufig indirekt mittels der „matched-guise“-Methode (Lambert et al. 1960), womit äußerst konsistente Befunde erbracht werden konnten, wie etwa, dass STANDARD-Sprecher/inne/n vor allem status-, NONSTANDARD-Sprecher/inne/n hingegen vor allem solidaritätsbezogene Eigenschaften zugewiesen werden (vgl. für Österreich z. B. Soukup 2009; zu Erklärungsansätzen Schoel \& Stahlberg 2012).

In den letzten Jahren ist jedoch vermehrte Kritik an der (vermeintlichen) Kontextlosigkeit und Stabilität beim gängigen Einstellungskonzept aufgekommen. Das führte zu einer Modifikation des Einstellungsbegriffs im Rahmen einer „sozial-konstruktivistische[n] Perspektive“ (Cuonz 2014: 32). Hierbei werden Einstellungen als soziale Konstrukte aufgefasst, die in Interaktionen laufend entstehen, modifiziert oder stabilisiert werden - die Folge war eine Öffnung der Einstellungsforschung hin zu qualitativen Methoden wie der Gesprächsanalyse (vgl. Liebscher \& Dailey-O’Cain 2009). Zugleich näherte sich der Einstellungsbegriff dadurch (noch) stärker dem Ideologiebegriff an (vgl. bspw. Soukup 2009: 88-89; zur Ähnlichkeit beider Konzepte etwa Garrett, Coupland \& Williams 2003). Der Begriff IDEOLOGIE, der vorrangig in sprachanthropologischen Paradigmen verwendet wird, bezeichnet dabei diskursiv erzeugte „conceptual schemes“ (Irvine \& Gal 2000: 35). Ideologien liefern „Just So Stories“, „characteristically by the way that they constitute rationalizing, systematizing, indeed most importantly naturalizing schemata that ,explain" the indexical value of signs“ (Silverstein 1992: 316). Wie Einstellungen sind Ideologien sozial erlernt dabei prägen sie das Verhalten der Sprecher/innen (vgl. Errington 1999: 115; Kroskrity 2009: 505) ebenso wie auch ihre Bewertungen (vgl. Cavanaugh 2013) bzw. das Denken und den Diskurs über Sprache (vgl. Coupland \& Jaworski 2004: 36). Die Spannweite von Ideologien ist groß und reicht „from seemingly neutral ,cultural conceptions" to strategies for maintaining social power“ (Woolard 1992: 239). Sprachideologien sind dabei häufig mit außersprachlichen Kategorien und Institutionen verwoben (wie Nation, Rasse, Geschlecht; vgl. Woolard \& Schieffelin 1994: 55-56). In diesem Sinne verknüpft bspw. die Ideologie des „homogeneism“ (Elspaß \& Niehaus 2014: 48) eine homogene STANDARDSPRACHE mit einer homogenen Gesellschaft.

Sowohl Einstellungen als auch Ideologien sind vielfach normativ - sie gehen mit Handlungsbewertungen und -erwartungen einher. Damit überlappen sie sich mit dem Normkonzept. Charakteristisch für NORMEN ist jedoch ihr klarer Handlungsbezug. Normen beruhen auf sozial geteilten Erwartungen zum er- 
laubten, verbotenen oder präferierten Handeln. Werden sie nicht befolgt, so ruft dies oft wertende Reaktionen bzw. Sanktionen hervor (vgl. zum Normbegriff ausführlich Bartsch 1987; Gloy 1975, 2004; Vergeiner 2021). Voraussetzung für die normbezogenen Erwartungen und Wertungen ist, dass Sprecher/innen Normvorstellungen haben, für die ein Geltungsanspruch kennzeichnend ist (der jedoch von faktischer Geltung zu unterscheiden ist, vgl. Hundt 2009: 118). Zur Herstellung und Stützung dieser Geltung werden Normen häufig im Rahmen von normativen Systemen mit anderen normativen Entitäten (z. B. auch Einstellungen, Ideologien) verbunden (vgl. Berger \& Luckmann 2016). Eine sehr wichtige Rolle spielen dabei auch Werte und Prinzipien - ähnlich wie Normen dienen sie zur Handlungsorientierung in sozialen Gruppen, schreiben dabei aber keine konkreten Handlungen, sondern vielmehr sein-sollende Zustände vor (vgl. Gloy 1975: 33).

Was sprachbezogene Einstellungen, Ideologien, Normen, Werte und Prinzipien verbindet, ist, dass sie letztlich (auch) auf die Laiensicht auf sprachliche Phänomene abzielen - wenn auch mit unterschiedlichen Schwerpunktsetzungen und wissenschaftsgeschichtlichem Hintergrund. Die Laiensicht auf Varietäten wird heutzutage zunehmend im Rahmen der Wahrnehmungsdialektologie zusammengefasst. Entstanden als folk linguistics beschäftigte sie sich anfangs vor allem mit der PERZEPTION von Laien auf DiALEKT-Grenzen und die (Un-)Ähnlichkeit von DiALEKTEN (vgl. bspw. Preston 2009). Gegenwärtig wird als Aufgabe der Wahrnehmungsdialektologie indes vielfach die allgemeine „Beschreibung kognitiver Strukturen des Alltagswissens“ gesehen (Anders 2010: 3; vgl. auch Hundt, Palliwoda \& Schröder 2015: 385-386 sowie kritisch bspw. Krefeld \& Pustka 2010: 13-14). Dies schließt Untersuchungen zur Laiensicht auf die Strukturierung und Verbreitung sprachlicher Varietäten ebenso ein wie varietätenbezogene Attribuierungen, Assoziationen und Einstellungen (vgl. bspw. Hundt, Palliwoda \& Schröder 2015: 369; Purschke \& Stoeckle 2019). Als Überbegriff für all diese zusammenhängenden Phänomene bietet sich der Ausdruck „Sprachkonzeption“ an (vgl. z. B. Hundt, Palliwoda \& Schröder 2015: 369). Im Rahmen des vorliegenden Aufsatzes sollen solche Varietätenkonzeptionen fokussiert werden, ohne den Forschungsgegenstand näher auf die weiter oben diskutierten Einzelphänomene festlegen zu wollen. Dies geschieht, weil, wie gezeigt, die Begriffe oftmals nicht abgrenzbar sind, sondern vielmehr zusammenhängen. Im Folgenden sollen laienlinguistische Sprachkonzeptionen in Hinblick auf drei Aspekte genauer betrachtet werden:

1. was die untersuchten Gewährspersonen (= GP) unter den jeweiligen Varietätenbegriffen verstehen, 
2. wie die GP die jeweiligen Varietäten und ihre Verwendung im Rahmen eines Bildungskontextes jeweils bewerten und

3. wann die Varietäten in diesem Bereich den GP folgend Verwendung finden sollen.

$\mathrm{Zu}$ beachten ist, dass wir den Laienbegriff in der vorliegenden Untersuchung sehr weit definieren - wir zählen als Laienäußerungen alle varietätenbezogenen Aussagen, die aus einer nicht-wissenschaftlichen Perspektive geäußert werden, auch wenn sie von GP stammen, die sich beruflich mit Sprache beschäftigen und/oder eine entsprechende Ausbildung aufweisen (z. B. Deutschlehrer/innen in der Schule oder Philolog/inn/en an der Universität). Wir tun dies, weil auch diese GP eine Alltagssicht auf Variation haben, die zwar beeinflusst von ihrem ,Expertenwissen' sein mag, aber nichtsdestoweniger nicht damit zusammenfallen dürfte. Auf welchen Daten und Methoden die Untersuchung beruht, wird im nächsten Abschnitt ausführlicher thematisiert.

\section{Daten und Methoden}

In der vorliegenden Studie wird ein Vergleich zwischen Varietätenkonzeptionen an sekundären und tertiären Bildungsinstitutionen in Österreich unternommen. Die analysierten und gegenübergestellten Befragungsdaten stammen zum einen aus dem Teilprojekt Wahrnehmungen von und Einstellungen zu Varietäten und Sprachen an österreichischen Schulen des SFB Deutsch in Österreich (= DiÖ) und zum anderen aus dem Projekt Verknüpfte Analyse von Mehrsprachigkeiten am Beispiel der Universität Salzburg (= VAMUS) (s. o. Anm. 2 und 3). Beide Projekte verbindet das Ziel, Perzeptionen und Attitüden zur Varietätenverwendung in Bildungskontexten zu untersuchen, wobei der Erhebungsschwerpunkt jeweils in Westösterreich liegt. Tabelle 1 gibt einen Überblick über das Datenmaterial beider Projekte, soweit es für die vorliegende Studie herangezogen wurde. ${ }^{4}$ Die

\footnotetext{
4 Die Datenkorpora von DiÖ wie VAMUS beinhalten jeweils auch Fragebogendaten, die aufgrund des Fokus auf qualitative Daten hier ausgespart bleiben (vgl. zu diesen Daten auch Vergeiner et al. 2019). Ausgeklammert werden weiters die Sprachgebrauchsaufnahmen beider Projekte, die nur indirekt Aufschluss über Varietätenkonzepte geben. Da der Untersuchungsschwerpunkt auf Salzburg und Tirol liegt, werden außerdem die Daten des DiÖ-Teilprojekts aus den Bundesländern Vorarlberg und Wien ausgespart.
} 
aus halbstrukturierten Interviews ${ }^{5}$ gewonnenen Daten wurden standardorthografisch transkribiert ${ }^{6}$ und inhaltsanalytisch ausgewertet.

Tab. 1: Untersuchtes Datenmaterial.

\begin{tabular}{|c|c|c|}
\hline Projektkorpora & DiÖ & VAMUS \\
\hline Erhebungszeitraum & $2017 / 2018$ & $2014 / 2015$ \\
\hline Untersuchungsort(e) & $\begin{array}{l}\text { vier berufsbildende mittlere und } \\
\text { höhere Schulen in den Bundes- } \\
\text { ländern Salzburg und Tirol }\end{array}$ & $\begin{array}{l}\text { eine mittelgroße öffentliche } \\
\text { Universität in Grenznähe zu } \\
\text { Deutschland (Universität Salz- } \\
\text { burg) }\end{array}$ \\
\hline GP & $\begin{array}{l}\text { Lehrer/innen aller Fächer und } \\
\text { Schüler/innen der } 10 \text {. Schulstu- } \\
\text { fe aus } 4 \text { Klassen ( } 50 \% \text { städ- } \\
\text { tisch; } 50 \% \text { maturaführend); } \\
\text { großteils aus der unmittelbaren } \\
\text { Region stammend; bei Schü- } \\
\text { ler/inne/n etwas mehr als die } \\
\text { Hälfte Sprecher/innen anderer } \\
\text { Erstsprachen (vor allem Tür- } \\
\text { kisch und die BKS-Sprachen) }\end{array}$ & $\begin{array}{l}\text { Lehrende und Studierende an } \\
\text { allen Fakultäten; jeweils bedeu- } \\
\text { tender Anteil von GP aus } \\
\text { Deutschland sowie Spre- } \\
\text { cher/innen mit anderer Erstspra- } \\
\text { che (vor allem DaF-, zum Teil } \\
\text { aber auch DaZ-Sprecher/innen) }\end{array}$ \\
\hline GP-Zahl in den & Lehrer/innen: 22 & Lehrende: 36 \\
\hline Interviews & Schüler/innen: 140 & Studierende: 66 \\
\hline
\end{tabular}

Wie Tabelle 1 zeigt, lässt sich das Datenmaterial von DiÖ und VAMUS nicht nur wegen der Befragungsmethode, sondern auch wegen ähnlicher Erhebungsräume, -zeitpunkte und ähnlich großer GP-Zahlen $(>100)$ gut vergleichen. Es bestehen allerdings gewisse Unterschiede bei den befragten Gruppen: Während im DiÖ-Korpus mehrere Untersuchungsorte berücksichtigt werden (je eine städtische und eine ländliche Schule in Salzburg und Tirol), beschränkt sich VAMUS auf nur einen Standort. Gleichwohl ist bei der Gruppe der universitären GP entsprechend dem größeren universitären Einzugsgebiet - eine größere Heterogenität gegeben: Anders als im Schulkontext kommen vermehrt Sprecher/innen vor, die nicht in Österreich bzw. im deutschsprachigen Raum aufgewachsen sind. Ein weiterer Unterschied besteht in der Altersverteilung, die sich bei den

5 Die Interviewleitfäden enthielten Fragen zu den Sprachbiographien der GP, zum (wahrgenommenen) Sprachgebrauch in den Institutionen sowie zu Spracheinstellungen.

6 Bei der Überführung in den STANDARD wurden auch Merkmale der GESPROCHENEN SPRACHE wie Rückversicherungs- und Häsitationssignale ausgespart. 
Schüler/inne/n im Gegensatz zu den anderen GP-Gruppen als weitestgehend homogen (16-17 Jahre) beschreiben lässt.

\section{Ergebnisse}

In diesem Abschnitt werden die Ergebnisse der Untersuchung präsentiert. Dabei wird zunächst auf die Befunde zur Universität, anschließend jene zur Schule eingegangen. Jeweils erfolgt zunächst eine Analyse der Konzeptionen von STANDARD, anschließend der von NONSTANDARD.

Aus Darstellungsgründen werden in der Folge nur exemplarisch Interviewäußerungen wiedergegeben. Bei der Zitation verweisen die Zahlen auf die Zeitfenster in den Aufnahmen. Zur Wahrung ihrer Anonymität werden die GP mit Siglen belegt. Sowohl bei den GP aus der Schule als auch bei denen aus der Universität steht der erste Buchstabe dabei für die GP-Gruppe (S = Studierende(r) bzw. Schüler/in); L = Lehrende(r)). Bei den GP aus der Universität stellen die Folgebuchstaben pseudonymisierte Initialen dar (z. B. „SIH“). Bei den GP aus den Schulen setzen sich die Folgezeichen zum einen aus Buchstaben, die den Schulstandort kennzeichnen, zum anderen aus Zahlen, die den einzelnen Proband/inn/en zugewiesen wurden, zusammen (z. B. „SA3“).

\subsection{Universität}

\subsubsection{Konzeptionen von StANDARD}

Universitäten gelten häufig als reine Domänen des STANDARDS; in ihnen werde, so die verbreitete Ansicht, nur die STANDARDSPRACHE gebraucht (vgl. Dannerer 2018: 169, 174-175). Im VAMUS-Korpus zeigt sich tatsächlich, dass in vielen universitären Situationen die Verwendung der STANDARDSPRACHE erwartet wird. Dies gilt vor allem für Prüfungen, den Sprachgebrauch von und mit Lehrenden sowie studentische Präsentationen. Diese Normvorstellungen wie auch die Rechtfertigungen dafür deuten auf eine Konzeption der STANDARDSPRACHE als formelle, distanzsprachliche Varietät hin. So meint etwa LIH, wenn „,[du] offiziell [...] als Lehrender vorne stehst, hast natürlich eine gewisse Rolle [...] und das ist schon verbunden mit der Standardsprache“ (LIH 26:28-26:42). Auf die Universität allgemein bezogen betont auch LUJ, er sei ein „Verfechter, dass man die Hochsprache sprechen sollte, gerade wenn es um offizielle Dinge geht, auch im Studium“ (01:36-02:01). Mit seiner distanzsprachlichen Funktion geht einher, 
dass der Gebrauch des STANDARDS mit Höflichkeit und Respekt assoziiert wird. Vor allem in der Kommunikation mit Lehrenden halten Studierende den STANDARD daher für angemessen. So ist etwa SIC überzeugt, es gebe ein „Niveau an Sprache, Standardsprache“, das für einen „respektvolle[n] Umgang“ mit Lehrenden unabdingbar sei; man rede mit ihnen „halt natürlich nicht so wie mit [...] Freunden“ (32:44-33:14).

Neben der Konzeption, dass die STANDARDSPRACHE diejenige Varietät ist, mit der man dem Gegenüber in besonderer Weise Höflichkeit und Respekt zum Ausdruck bringt, wird sie auch häufig mit Korrektheit assoziiert. Dahinter mag die Ideologie stehen, dass (nur) die STANDARDSPRACHE korrekt sei. Für etliche GP, wie etwa SUI, ist die STANDARDSPRACHE das „korrekte Deutsch, das auch in geschriebener Sprache existiert und bestimmte grammatikalische Regeln hat und Normen“ (SUI 19:12-19:32). Gerade die Verbindung zwischen SCHRIFTLICHKEIT NORMIERTHEIT - STANDARDSPRACHLICHKEIT ist an der Universität weit verbreitet. Der STANDARD sei „halt dann wirklich korrekt“, insofern man spricht, wie man schreibt, urteilt etwa SIH (27:21-27:28). Diese Gleichsetzungen sind wohl ursächlich dafür, dass STANDARD-Sprecher/inne/n auch eine höhere Kompetenz und Professionalität unterstellt wird. LOB bspw. ist „nicht dafür, dass wir [...] im Lehrbetrieb Umgangssprache sprechen [...]. Die Professionalität hängt auch ein bisschen daran, dass man gehobeneres Deutsch spricht“. Nicht nur auf sie wirken bspw. Sprecher/innen aus dem Norden Deutschlands allein wegen ihrer STANDARDNAHEN Sprechweise „unglaublich kompetent“ (16:18-17:37). In Verbindung mit solchen Einstellungen steht, dass die STANDARDSPRACHE als bildungssprachliche Varietät konzipiert wird: „Ich glaube schon [...], dass es erwünscht ist, wenn man halt schöner spricht [...], es klingt irgendwie gebildeter“, denkt etwa SUG (24:45-25:15). Auch SOE bekundet, in Referaten sei STANDARD zu verwenden, weil es „einfach seriöser klingt“ - ,wissenschaftliche Sachen, die sind halt doch irgendwie immer eher bisschen steif und das ist für mich auch die Standardsprache“ (21:34-22:42).

Eine besonders häufig vorgebrachte Zuschreibung der STANDARDSPRACHE ist ihre hohe Verständlichkeit. Im Allgemeinen wird die STANDARDSPRACHE als verständlicher und damit als angemessener für universitären Sprachgebrauch angesehen als NonSTANDARD. So ist etwa SIC dafür, „dass die Professoren selber darauf schauen bisschen, dass alle Hochdeutsch sprechen“ - NONSTANDARD würden nämlich „nicht alle verstehen“ (05:31-05:37). Als besonders unverständlich werden vom STANDARD abweichende Varietäten für Sprecher/innen mit anderer L1 als Deutsch angesehen. So erwartet etwa LOE, dass man wegen der „Internationalisierung unserer Universitäten“ und der „zahlreichen Incomings“ generell STANDARD verwenden sollte. „Dass sie unsere Sprache verste- 
hen“, sei ein „Argument der Höflichkeit“ (09:18-10:52). Bisweilen wird der Gebrauch der STANDARDSPRACHE auch in Gegenwart von Sprecher/inne/n aus Mittel- und Norddeutschland befürwortet, da auch diesen unterstellt wird, keinen bairisch-österreichischen NONSTANDARD zu verstehen: „wenn ich mit [...] jemandem aus Norddeutschland spreche, dann versteht er mich nicht, so wie wir zwei jetzt reden, oder hat seine Mühe nur, da muss ich mich anpassen und Hochdeutsch verwenden“, wertet etwa die Österreicherin SUH (19:39-19:51). ${ }^{7}$ Bundesdeutschen Sprecher/inne/n (vor allem aus dem Norden und der Mitte Deutschlands) wird an der Universität Salzburg allerdings nicht nur unterstellt, keinen regionalen NONSTANDARD zu verstehen; ihnen wird auch zugeschrieben, die ,echten“ bzw. ,besten“ STANDARD-Sprecher/innen zu sein: „sie sprechen natürlich schöner, akkurater, und nicht so schlampig wie wir Österreicher“, urteilt z. B. die Österreicherin VIA (01:52-02:01). Das ist nicht nur die Fremdwahrnehmung österreichischer GP, auch viele bundesdeutsche GP sind davon überzeugt. LUJ vertritt etwa die Meinung, dass er in Österreich „eigentlich noch nie auf einen gestoßen [ist], der Hochdeutsch spricht“ (17:10-17:14), höchstens auf Sprecher/innen, die „Pseudohochdeutsch“ verwenden. Was er als Norddeutscher spreche, „komme dem Hochdeutschen wahrscheinlich relativ nahe“, die STANDARDORIENTIERTE Sprechweise in Österreich sei hingegen „vom Hochdeutschen relativ weit entfernt und hat noch immer ganz deutliche dialektische [sic] Einfärbungen“ (03:30-03:50).

STANDARDSPRACHLICHKEIT wird oft damit gleichgesetzt, dass eine Sprechweise frei von regionalen Merkmalen sei. ${ }^{8}$ Dabei werden Merkmale als STANDARDkonstitutiv genannt, die auf ein sehr rigides, monozentrisches Konzept von STANDARD hinweisen - die Bayerin SOE bspw. ist davon überzeugt, sie könne „einfach nicht richtig Hochdeutsch sprechen [...] ich sage zum Beispiel [jpoett] ${ }^{9}$ und nicht [jpэьt], oder im Bayrischen gibt es kein summendes [z] [...], oder das [v] ist ganz

7 Auffällig ist, dass die Sprechweise im Ausschnitt nicht sonderlich DIALEKTAL ist, vgl. die Übertragung der Transkription in GAT2: ,we_wenn i mit am (-) m_mit am (.) mit jemanden aus NORDdeutschland sprech? | dann verSTEHT er mi ned, | so wie WIR zwa jetzt reden;=JA? | ${ }^{\mathrm{h}}$ äh oder (.) hot seine MÜhe nur;=JO?=| =do muss i mi ONpassn." Zu erwähnen ist, dass nicht alle GP aus der Ansicht, dass Bundesdeutsche NONSTANDARD nicht verstehen, folgern, es sollte STANDARD mit ihnen gesprochen werden - vielmehr sind sie der Ansicht, Deutsche sollten NONSTANDARD verstehen lernen (vgl. dazu Vergeiner 2020).

8 Dies führt etwa zu der paradoxen Situation, dass Sprecher/innen, die ein vermeintlich, von regionalen Merkmalen freies Deutsch' sprechen, in einer bestimmten Region verortet werden, z. B. ,in der mitte von deutschland“ (vgl. Beuge 2019: 161; auch 188, 227, 274f.).

9 Um Missverständnisse zu vermeiden, sei erwähnt, dass die Sprecherin hier natürlich eine völlig korrekte Ausspracheform vorbringt, diese aber für defizient hält. 
anders“ (23:23-23:41). Diese starre Auffassung des STANDARDS hat zur Folge, dass nicht nur Österreicher/inne/n, sondern bisweilen überhaupt den meisten oder allen Deutschsprachigen abgesprochen wird, eine (mündliche) STANDARDKOMPETENZ zu besitzen. LAF hält den GESPROCHENEN STANDARD etwa für eine „reine Kunstsprache“ - es gebe nur „Umgangssprache“ und „Österreichisch ist genauso [...] wenig Hochdeutsch wie jetzt irgendeine Umgangssprache in Deutschland“ (72:05-73:08). Andere GP sind indessen bereit, mehr Variation zu akzeptieren und vertreten Positionen, die eher einem GEBRAUCHSSTANDARD (vgl. Elspaß \& Kleiner 2019: 159-160) entsprechen - so charakterisiert etwa LED sein Deutsch zwar als „ein bisschen österreichisch gefärbt, aber grundsätzlich ist es die Hochsprache“, auch wenn er Strukturen gebraucht, „die man natürlich in Norddeutschland nicht verwenden würde“ (16:52-17:11). Insgesamt sind solche Konzeptionen aber eher selten - auch der tatsächliche Sprachgebrauch ist eher von einem am Monozentrismus und der Explizitlautung orientierten Konzept des STANDARDS geprägt (vgl. ausführlich Vergeiner 2021).

\subsubsection{Konzeptionen von NonStANDARD}

Auch wenn an der Universität vielfach der Gebrauch von STANDARD erwartet wird und die Konzeptionen von STANDARD generell eher positiv ausfallen, wird an der Universität nicht ausschließlich STANDARDSPRACHLICHKEIT propagiert. Etliche GP halten die Verwendung von DIALEKT und UMGANGSSPRACHE ${ }^{10}$ als informelle, eher nähesprachlichere Varietäten an der Universität für legitim, zumindest in gewissen Situationen wie spontanen Wortmeldungen oder Sprechstundengesprächen.

Dafür scheinen nicht zuletzt gewisse Einstellungen verantwortlich, die insbesondere DIALEKT mit Attributen wie Authentizität, Identität oder Spontanität verbinden. Dass DiALEKT an der Universität vorkomme, hält etwa SUK für „berechtigt“, denn „es macht das Ganze ein bisschen ungezwungen und lebendig, authentisch“ (20:48-20:58). Auch der Lehrende LAE bemerkt, dass durch die Verwendung von NONSTANDARD-Varietäten der Sprachgebrauch an der Universität „aufgelockert“ werde - dadurch komme man „wesentlich authentischer rüber“, sei „pointierter“ und „vom alltäglichen Umgang her“ sei die Kommuni-

10 Der durchaus umstrittene Begriff wird hier verwendet, da es sich bei vielen GP um eine Teilnehmerkategorie handelt (s. u.). Wir nutzen ihn als solchen, ohne damit eine diskrete Sprachschicht zwischen DIALEKT und STANDARD postulieren zu wollen (vgl. zur Diskussion bspw. Vergeiner 2019). 
kation dadurch „einfach [...] viel einfacher“ (26:20-26:50). Äußerungen wie diese verweisen einerseits auf eine Ambivalenz der Konzeptionen von STANDARD, insofern Zuschreibungen wie Formalität und Distanziertheit auch negativ wahrgenommen werden können. Sie belegen andererseits aber auch die hohe Loyalität, die etliche GP an der Universität gegenüber NONSTANDARD-sprachlichen Varietäten aufweisen - wie etwa SOE, die von sich behauptet, „immer Dialekt“ zu reden, komme sie sich doch beim Sprechen des STANDARDS „immer so gekünstelt vor“, es sei dann ihre „Stimme ganz anders und das bin dann einfach nicht mehr ich“. Sie wertet es daher positiv, dass sie an der Universität auch ihren DIALEKT sprechen dürfe (17:33-18:42).

Doch auch GP, die selbst keine DIALEKT-Sprecher/innen sind, verbinden mit dem DiALEKT vielfach positive Eigenschaften. Auffallend oft werden dabei ästhetische Urteile gefällt: „Mir [...] gefallen Dialekte“, betont etwa SOF, der weiter ausführt, die Verwendung von DIALEKT bringe „ein bisschen Pepp“ und „Spannung“ in Lehrveranstaltungen, während es ohne Sprachvariation „langweilig“ sei (02:33-03:02). Besonders häufig wird auch darauf verwiesen, dass es „gut“ sei, wenn man „hört, wo wer herkommt“ (SUA 15:31-15:48). Und während einige GP durch den Gebrauch von DiALEKT zumindest „keine Nachteile“ sehen, „solange alle Anwesenden zurechtkommen“ - schließlich sei „Sprache“ nur „ein Mittel der Verständigung“ (LOH 03:37-04:49) - tragen DiALEKTE nach Ansicht anderer GP zur sprachlichen Vielfalt an der Universität bei. Das wird als positiver Wert gesehen: Z. B. sollte SOL zufolge „nicht jeder sprachlich so zurecht gedengelt werden [...] dass alles uniform klingt“ (33:48-33:56) - „Diversität“ sei schließlich „immer etwas Gutes“ (38:47-38:56). Ein Topos, der in diesem Zusammenhang immer wieder angesprochen wird, ist der (vermeintliche) Schwund des DiALEKTS: SUK bspw. befürchtet, dass manche Personen DiALEKTE „ausrotten“ und „aussortieren“ wollen; das sei „schade“, habe der DIALEKT doch einen „gewissen Wert“, gerade im „Alpenraum“, der DIALEKTAL „so extrem vielschichtig ist“ und wo DIALEKTE auch für die kulturelle Identität „prägend sind“; um den DiALEKT-Abbau zu verhindern, sollten DIALEKTE auch an der Universität Verwendung finden (31:42-33:38).

Allerdings treten an der Universität auch negative Einstellungen zu und Wertungen von NONSTANDARD-Varietäten zutage. Im Vergleich zur STANDARDSPRACHE wird insbesondere der DIALEKT als potentielles Verständnishindernis dargestellt (s. o.), außerdem sei es so, wie etwa LOC bemerkt, dass viele „Intelligenz nicht mit Dialekt“ verbinden (12:41-12:59). Damit zusammenhängend wird NONSTANDARD oftmals als inkorrekte und falsche Form der Sprachverwendung begriffen. So charakterisiert etwa SIH den Begriff des DIALEKTS wie folgt: „Dialekt ist mehr so, ja, ich weiß eigentlich eh, wie es richtig ist, aber es fällt mir unheimlich 
schwer, das im Sprachgebrauch anzuwenden“ (32:24-32:33). Andere GP vertreten zwar sehr wohl die Ansicht, dass auch der DIALEKT Regeln und Normen habe, obgleich diese vom STANDARD abweichen. So ist etwa nach SIC im STANDARD „Vieles nicht richtig“, was „im Dialekt einfach richtig ist [...], er [= der DiALEKT] hat einfach eigene Regeln und eine eigene Grammatik“ (37:28-37:34). Nichtsdestoweniger halten viele GP den DIALEKT zumindest in Bezug auf die wissenschaftliche Sprachverwendung jedoch für nicht ausreichend ausgebaut. „Einen wissenschaftlichen Vortrag“, ist etwa LEA überzeugt, „,komplett im Dialekt, das geht ja kaum [...], Dialekt hat ja einfach nicht diesen funktionalen Geltungsbereich“ (05:40-05:50). Auch SUH äußert z. B. die Ansicht, im DiALEKT könne man nicht „kurz und prägnant und klar formulieren“, weshalb man in Prüfungen STANDARD nutzen solle (19:51-20:25).

Häufiger vorgebracht wird auch der (Irr-)Glaube, der DIALEKT sei ein Resultat des Sprachverfalls oder eine, wie etwa SAB äußert, „verzerrte Form [...] von der Standardsprache“ (29:41-29:49). Eine besonders drastische Ansicht ist, vermehrter Gebrauch des DIALEKTS sei schädlich, wie sie etwa SEG vertritt; sie berichtet in diesem Zusammenhang von einer Lehrveranstaltung in „Stimmbildung“, wo von einer externen Lehrenden (einer „Opernsängerin aus München“) vermittelt worden sei, der Gebrauch von DIALEKT schädige die Stimmbänder und wenn jemand „ständig Dialekt spricht“, könne der-/diejenige die „Stimme verlieren“(36:42-38:00).

Negative Konzeptionen finden sich häufig auch von der UMGANGSSPRACHE. Von manchen GP wird sie sogar als noch weniger angebracht für den Gebrauch an der Universität eingestuft als etwa der DiALEKT; so meint etwa LAC, man habe „weniger ein Problem“, dass das „Sprachniveau zu niedrig wird [...], wenn man Dialekt spricht [...] als wenn man Umgangssprache spricht“ (07:11-07:22). Solche Konzeptionen legen nahe, dass die UMGANGSSPRACHE nicht von allen GP als Sprechweise ,zwischen' DIALEKT und STANDARD begriffen wird - auch wenn viele GP dies tun -, sondern als SozIOLEKT: „Umgangssprache ist halt, immer so viel Englisch drinnen [...], das ist halt Jugendsprache“ definiert den Begriff etwa SIH (27:28-27:43). Andere GP bekunden, das Konzept überhaupt nicht zu kennen oder sie rücken es in die Nähe der STANDARDSPRACHE. So etwa LEG, wenn er meint, er habe eigentlich „immer in Dialekt und Schriftsprache gedacht“ durch die Fragestellungen bei der VAMUS-Erhebung sei er jedoch darauf gekommen, dass es „dreigeteilt“ ist, sei doch ein „große[r] Unterschied zwischen wirklich Schriftdeutsch“ und dem, „was ein österreichischer Fernsehsprecher redet“ (06:08-06:31). Dass nach LEGs Ansicht selbst professionelle Sprecher/innen NONSTANDARD in Form einer UMGANGSSPRACHE verwenden, belegt 
nicht zuletzt die eher monozentrische Auffassung des STANDARDS vieler GP, von welcher oben die Rede war.

\subsection{Schule}

Auch an der Schule existieren unterschiedliche Varietätenkonzeptionen, die z. T. mit denen an der Universität übereinstimmen, sich z. T. auch unterscheiden. In der Folge werden zunächst die STANDARD-, dann die NoNSTANDARDKonzepte in der Schule besprochen und mit der Universität verglichen.

\subsubsection{Konzeptionen von STANDARD}

Betrachtet man die Konzepte von STANDARD an der Schule, fällt zunächst ein Unterschied zwischen Stadt und Land auf: Der STANDARD wird von den meisten GP aus dem städtischen Raum als Sprache des täglichen Umgangs definiert. So ist es Schülerin SA47 zufolge eine „komplett normale Sprache“, die in der „Alltagskommunikation“ gesprochen wird (12:14-13:21). SA10 bestätigt das dahingehend, als diese Varietät etwas sei, „das alle reden“ und „das am Ende des Tages von allen verstanden wird“ (19:35-20:21). SA21 geht noch weiter, indem sie meint, dass die STANDARDSPRACHE diejenige Varietät sei, die „in Österreich allgemein gesprochen“ werde und „im täglichen Austausch“ die wichtigste Kommunikationsform darstelle - sei es nun „im Arbeitsleben“ oder „in der Freizeit“ (15:50-19:22). Auch Schüler SA23 ist der Meinung, dass der STANDARD „eine vorgegebene Sprache für alles“ sei, die auch allenthalben genutzt werden sollte. Ihm zufolge habe es „nur Vorteile“, dass in Salzburg-Stadt keine DIALEKTE gesprochen würden. Es gebe keine Verständnisprobleme und dadurch sei die Kommunikation „viel unkomplizierter“ (20:21-21:15). Interessanterweise wird der STANDARD von den Schüler/inne/n in Salzburg-Stadt viel deutlicher als „Alltagssprache“ definiert als von ihren Lehrer/inne/n, die dabei wesentlich vager bleiben. Trotzdem ist der STANDARD auch für etliche städtische Lehrpersonen eine Art GEBRAUCHSSTANDARD, der in den meisten Interaktionssituationen angemessen ist und den Lehrenden zufolge dabei auch Potential für Variation aufweist.

Nicht überall gilt der STANDARD jedoch als „Sprache des täglichen Umgangs“ - vor allem in stark DIALEKTAL geprägten, ländlichen Regionen wird er im Gegenteil als „eine Art Fremdsprache“ verstanden, wie es etwa LC4 ausdrückt, wobei er als solche zum Teil „nur wenig Ähnlichkeiten“ mit dem lokalen DiALEKT aufweise. Dennoch sei es laut LC4 vor allem in Hinblick auf die (mündlichen) Ma- 
turaprüfungen notwendig, dass die Schüler/innen auch mit dem HocHDEUTSCHEN konfrontiert werden (19:18-21:08). Auch andere GP konzipieren den STANDARD als Art Fremdsprache - wie beim Fremdspracherwerb erfolge laut LC2 etwa der Übergang vom DiALEKT in die STANDARDSPRACHE „prozesshaft“. Nach und nach könne die STANDARD-Kompetenz aufgebaut und dieser in verschiedenen Situationen eingesetzt werden (17:33-19:09).

Auch wenn der STANDARD zumindest in ländlichen Regionen als Art Fremdsprache wahrgenommen wird, wird er nichtsdestoweniger an allen Schulstandardorten oft - wie an der Universität (vgl. Abschnitt 4.1.1) - als korrektes Deutsch vorgestellt. SC22 vertritt bspw. die Ansicht, dass das HocHDEUTSCHE teilweise einen „ganz anderen Wortschatz“ als der DiALEKT umfasse, wobei HocHDEUTSCH im Gegensatz zum DIALEKT auch „grammatikalisch korrekt“ sei. Das mache eine Verständigung außerhalb der eigenen Region schwierig. Schon allein deshalb sei „das Erlernen der Standardsprache notwendig“ (15:22-15:47). Auch an der Schule wird von den GP der STANDARD gemeinhin als „klarer“, „deutlicher“ und auch ,gebildeter“ angesehen, wie etwa SA45 meint. Ihr zufolge versuche man mit HocHDEUTSCH „etwas darzustellen“, „sich gut zu positionieren“ und dadurch auch „ernster genommen zu werden“ (17:03-17:55). Ähnlich argumentiert SA52. Für sie zeugt HocHDEUTSCH von „Respekt, von Hochachtung“. Indem man STANDARD spricht, zeige man, dass man das Gegenüber „akzeptiert“ und „schätzt“. Insofern SA52 den STANDARD mit der Sprache Goethes vergleicht, wird auch deutlich, dass sie HocHDEUTSCH als Sprache der Gebildeten und der Bildung ansieht (15:22-16:03). Für SA2 ist die STANDARDSPRACHE dabei auch eine Sprechweise, die „so vorgegeben ist“ und „bestimmten Regeln entspricht, an die man sich halten muss“ (13:28-14:50). SA6 zufolge ist es wichtig, „die Wörter so auszusprechen, wie sie sind“. Erst dann sei es „richtiges Hochdeutsch“. Obwohl es für diese GP „nicht schlimm“ ist, wenn dazwischen ein paar Wörter im DIALEKT vorkommen, zeuge das trotzdem für ,weniger Intelligenz“ und sei auch „nicht so schön“ (13:58-14:32). Dass STANDARD mit Bildung, Respekt und Höflichkeit verbunden wird, entspricht der Sicht, die an der Universität vorherrscht (vgl. Abschnitt 4.1.1).

Auch in Regionen, in denen der DIALEKT die unumstrittene Alltagsvarietät ist, stellt die STANDARDSPRACHE die Varietät mit dem höchsten Prestige dar, die mit der Assoziation hoher Bildung einhergeht. So bezeichnet SC5 den STANDARD als „höher, gebildeter“ (12:05-12:35). SC25 unterstreicht diese Meinung, indem er die STANDARDSPRACHE als „eine gehobene Weise von Dialekt“ bezeichnet, die „gepflogener und schöner“ ist. Für ihn steht sie in unmittelbarem Zusammenhang mit „Vornehmheit“. STANDARD sei eine „saubere Sprache, ohne Fehler, in dem man alles klar ausdrücken“ könne. Vor allem „die Gebildeten“ würden 
HocHDEUTSCH sprechen, während in der Familie oder mit Freunden doch eher der DIALEKT im Vordergrund stehe. Vor Leuten, die die STANDARDSPRACHE im täglichen Leben benutzen, habe er „großen Respekt“ (11:05-12:01). Aus einem ähnlichen Grund hält es auch SA20 für unangebracht, in der Schule DIALEKT zu sprechen. HocHDEUTSCH sei die „Höflichkeitsform“, die „förmlicher“ als der DiALEKT sei und „die man außerhalb der Familie oder des Freundeskreises“ immer sprechen sollte (07:52-09:19).

Weil der STANDARD als förmlichere Sprechweise konzipiert wird, ist der ausschließliche Gebrauch des STANDARDS sowohl Lehrer/inne/n als auch Schüler/inne/n zufolge in bestimmten schulischen Situationen anzustreben. Dies betrifft - ähnlich wie an der Universität (vgl. Abschnitt 4.1.1) - vor allem die fachlichen Teile bei Referaten (bei Lehrer/inne/n teilweise auch Fachvorträgen) oder Prüfungssituationen. Schüler SA1 betont in diesem Sinne, dass es sich „einfach nicht gehört, bei Referaten Dialekt zu sprechen“ (06:58-07:03). Eine ähnliche Ansicht vertritt der Lehrer LC3, der beteuert, dass „dialektal gefärbte“ Sprache bei einer Abschlussprüfung „natürlich nicht geht“ (04:28-04:55). Als oberstes Ziel wird dabei oft die mündliche Abschlussprüfung (d. h. Matura- bzw. Fachprüfung) genannt. Auch in Hinblick auf ein Universitätsstudium sei, so sind die schulischen GP nicht zu Unrecht (vgl. Abschnitt 4.1.1) überzeugt, eine gewisse Versiertheit in der STANDARDSPRACHE notwendig. Das ist dann auch der Grund für LA3, ihre Schüler/innen auf eine solche Versiertheit vorzubereiten; schließlich sei sie selbst, im Gegensatz zu „deutschen oder ostösterreichischen“ Kommiliton/inn/en, die sich die adäquate Sprache „nicht zuerst überlegen mussten“, an der Universität oft in einen „sprachlichen Notstand“ geraten, weil die „Grammatik und Lexik“, die sie aus dem DIALEKT gewohnt war, für formelle Situationen nicht mehr passend schien. Um den Lernenden solche Schwierigkeiten zu ersparen, versuche sie, ihnen „anhand eigener Erfahrungen Empfehlungen zu geben“ und in Vorbereitung auf das Studium mit ihnen die STANDARDVARIETÄT zu trainieren (09:37-10:18).

Dass die Schule den Erwerb von STANDARD auch in Hinblick auf das spätere Berufsleben forcieren müsse, kommt häufiger vor. Dabei scheint das Erlernen von HochDEUTSCH in der Schule als überregionale Varietät von einem Großteil der GP als Vorteil für das spätere Berufsleben eingeschätzt zu werden. Verwiesen wird im Zuge dessen insbesondere auf Wirtschaftsberufe oder Berufe, die ein Studium voraussetzen, wie etwa der Arzt- oder Lehrberuf. SC25 zufolge werde man in solchen Berufen „,besser wahrgenommen“ und sei dadurch „erfolgreicher“, wenn man sich „gut auf Hochdeutsch artikulieren“ könne (21:1421:57). Ein ähnliches Argument bemüht SC41, die der Meinung ist, dass die STANDARDSPRACHE „das Um und Auf im Geschäftsleben“ sei (19:18-21:03). Auch 
Bewerbungsgespräche werden in diesem Zusammenhang öfter genannt. In ihnen spricht etwa SC4 „eher Hochdeutsch“, weil sie sich „gut ausdrücken“ will, außerdem ist der STANDARD für sie ein Mittel, um vom Gegenüber gut verstanden zu werden (03:30-04:05). Für LA2 ist es gleichsam in einer „globalisierten Welt“ Voraussetzung, dass man ,in einer Sprache spricht, wo man einen in Norddeutschland auch versteht.“ Gerade für ihren Schultyp sei das umso wichtiger, wo doch ein Großteil der Absolvent/inn/en zukünftig in wirtschaftlichen Betätigungsfeldern arbeiten werde (09:07-09:28). Dass STANDARD verwendet werden soll, um den Erwerb des STANDARDS zu forcieren, ist ein Aspekt, der an der Universität zwar auch belegbar ist, aber nicht so oft, da STANDARDKompetenzen dort vielfach erwartet werden (vgl. ausführlicher etwa Vergeiner et al. 2020).

Gerade in Hinblick auf das spätere Berufsleben zeigt sich, dass die GP an der Schule dem STANDARD nicht nur ein höheres Prestige, sondern auch eine bessere Verständlichkeit zuschreiben. Dieser Wert stellt für die meisten ein starkes Argument für die Orientierung am STANDARD dar. Zumindest für manche GP spricht dies auch für einen verstärkten Gebrauch von STANDARD in der Schule. Für die Lehrperson LG1 ist es z. B. explizit „erwünscht“, dass „Schriftsprache“ gesprochen werde. Ihr zufolge hätten vorwiegend Schüler/innen aus stark dialektal geprägten Regionen zwar anfangs oft Schwierigkeiten, sich „sprachlich umzustellen“; „erstrebenswert“ sei das aber allemal, zumal Lernende aus DIALEKTFERNEREN Gebieten oft Schwierigkeiten hätten, den starken DIALEKT zu verstehen (05:22-06:18). Auch die große Zahl an Schüler/inne/n mit Deutsch als Zweitsprache mache es SG5 zufolge notwendig, sich an die STANDARDSPRACHE anzunähern (15:18-15:56).

Auffällig an den schulischen Interviews im Vergleich zur Universität ist, dass manche GP zwar unterschiedslos von HOCHSPRACHE und STANDARDSPRACHE sprechen, dass andere GP jedoch zwischen beiden Begriffen sehr wohl differenzieren. Sie unterscheiden dabei zwischen STANDARD, der in Österreich als „höchste Varietät“ möglich ist, und НосHDEUTSCH. Letzteres sei eine Sprachform, die „rein in Deutschland“ gesprochen wird und für Österreicher/innen „gar nicht realisierbar“ sei. SA33 zufolge sei das aber auch nicht notwendig. Damit einher geht auch die Auffassung von einem eigenen ÖSTERREICHISCHEN STANDARD.

Für viele GP ist dieser ÖSTERREICHISCHE STANDARD dem DEUTSCHEN STANDARD indes untergeordnet im Sinne einer asymmetrischen Plurizentrik (vgl. Scharloth 2005 für eine ähnliche Situation in der Deutschschweiz). Trotz der Überzeugung, dass verschiedene Zentren existierten und ein Einheitsdeutsch nie erreicht werden könne, stellt ein solches den GP zufolge ein Ideal dar, das über allen anderen Varietäten steht. Das österreichische Deutsch, das innerhalb 
Österreichs die „höchste Sprachlage“ darstelle, wird dabei als inferior betrachtet. So ist nach Ansicht von LG2 das „deutsche Deutsch lupenreiner“ im Vergleich zum „holprigen“ österreichischen Deutsch, Deutsche wirkten „kompetenter“, „rhetorisch besser“ und seien viel eher in der Lage, etwas auf den Punkt zu bringen (07:22-08:23). Laut SA40 gibt es einen großen Unterscheid zwischen dem DEUTSCHEN und dem ÖSTERREICHISCHEN STANDARD. Bewusst sei ihr das erst geworden, als ihre vierjährige deutsche Cousine sie fragte, warum sie nicht „richtig Deutsch“ sprechen könne. Sie würde sie „nicht gut verstehen“, weil sie „so komisch“ rede (20:16-20:49).

SA52 zufolge würden in Österreich „die gleichen Wörter benutzt“ wie in Deutschland, diese würden aber „schlampiger ausgesprochen“. Im Gegensatz dazu klinge das HochDEUTSCHE „viel hochgestochener“ und sei „viel näher an der Rechtschreibung und an der Norm“ (15:33-16:25). Dass die Schreibung als Maßsta $b$ für STANDARDSPRACHLICHKEIT gesehen wird, zeigt sich generell auch bei anderen GP - STANDARDSPRACHLICH ist nach Meinung von SC37 „das Schriftliche“, was für die Alltagskommunikation „nicht relevant“ sei. Insofern gilt der STANDARD als „normierte und kodifizierte Schriftsprache“, wie sie in den Lehrbüchern verwendet wird (20:18-21:03). Laut SA12 ist HochDEUTSCH genau so, „wie man schreibt und formuliert“. Es sei in Bezug auf die Grammatik „genau so, wie man es in der Schule lernt“ (15:14-15:46). Auch für SC12 ist die STANDARDSPRACHE ebendas, was „,im Wörterbuch“ zu finden sei. Es sei ein „sehr sachliches und grammatikalisch korrektes Deutsch“, das in Österreich „die Amtssprache" darstelle und deshalb auch in der Schule gelernt werden müsse (23:30-24:45). Letztlich ist die Gleichsetzung von SCHRIFTLICHKEIT - NORMIERTHEIT

- STANDARDSPRACHLICHKEIT also an der Schule genauso wie an der Universität verbreitet. Dasselbe gilt - wenn auch etwas weniger stark - für die areale Verortung des STANDARDS in Deutschland.

\subsubsection{Konzeptionen von NonstandaRD}

Obwohl die STANDARDVARIETÄT eng mit dem schulischen Alltag verwoben zu sein scheint und gerade den Lehrpersonen zufolge unabdingbar in formelleren Situationen ist, wird oft für den Gebrauch von NONSTANDARD-Varietäten im Schulalltag argumentiert:

Analog zur Universität (vgl. Abschnitt 4.1.2) wird NONSTANDARD dabei vorwiegend mit Nähe, Heimat und Verwurzelung in Zusammenhang gebracht. Von SC6 als „emotionale Herzenssprache“ mit kleinräumiger Verbreitung definiert, zeichne er sich durch „lokale Sprachnuancen“ aus und unterscheide sich in 
„wesentlichen Elementen“ von der STANDARDSPRACHE. Insofern sei der DiALEKT stark mit „Heimat“ in Beziehung zu bringen und als solcher ein „wichtiger Bestandteil“ ihres Lebens (09:46-10:01). Für LC2 zeigt sich die regionale Verwurzelung in der Verwendung von DIALEKT, der für sie „die Wurzel“, die „wirkliche Muttersprache“, in der man „fast alles“, insbesondere aber „Emotionen und Gefühle“, viel besser ausdrücken könne (12:06-13:10). LC4 teilt diese Sichtweise, wobei sie den Stellenwert des DiALEKTS für die Jugendlichen hervorstreicht. Die regionale Varietät würde ihnen ein „gewisses Selbstbewusstsein“ geben, sie hätten das Gefühl, „irgendwo dazuzugehören“. Der DiALEKT habe demgemäß viel mit „Identität, mit Persönlichkeit“ zu tun. Durch die Sprache würde man sich „mit einem Gebiet identifizieren“. In ihrem Unterricht sei es ihr daher wichtig, dass die Schüler/innen „authentisch“ blieben und sich nicht „verstellten“ (15:14-15:23). Immer wieder wird, wie an der Universität (vgl. Abschnitt 4.1.2), dieser Zusammenhang von Identität und DIALEKT betont. LF5 zufolge betreffe er selbst Schüler/innen mit Migrationshintergrund: Sie wüssten oft nicht, welcher Nationalität sie sich zugehörig fühlen. Allein die regionale Varietät und die dadurch geschaffene Solidarität helfe ihnen, sich zu integrieren (07:18-08:52).

Das Zugeständnis an den als authentisch empfundenen NONSTANDARD wird an der Schule - und hier unterschieden sich Schule und Universität - auch öfter auf die pädagogische Beziehung zwischen Lehrenden und Lernenden bezogen, zumindest wenn es sich nicht um offizielle Situationen handelt. Für LA2 ist es das Kennzeichen einer „guten Beziehung“, wenn die Schüler/innen mit ihr im DIALEKT sprechen. Die Lernenden würden sich dadurch „wohl“ und „,angenommen“ fühlen, was für eine „gute Vertrauensbasis“ unabdingbar sei. In Prüfungssituationen müssten sie natürlich „ordentlich“ sprechen (05:10-06:04). An der Universität scheint die nähesprachliche Funktion von NONSTANDARD bei Lehrenden-Studierenden-Interaktionen weit weniger bedeutsam zu sein.

Das gilt auch für den didaktischen Wert, der dem DiALEKT an der Schule zugemessen wird, etwa beim Sprachenlernen: Für LF2 z. B. bildet der DiALEKT als „Muttersprache“ die Basis für den weiteren Varietäten- und Spracherwerb. In der regionalen Sprache seien „zusätzliche Informationen“ enthalten. „Über Jahrzehnte bzw. sogar Jahrhunderte entwickelt“, habe diese Varietät „eine gewisse Tradition“. Verstehe man das, „woher man kommt“, könne man auch „das große Ganze“ besser begreifen. Egal, ob man dann „gehobene Umgangssprache“, „Standard“ oder sogar eine „zweite oder dritte Fremdsprache“ lerne, mit dem DiALEKT als Basis habe man weit weniger Schwierigkeiten damit (14:2215:35).

Im Unterschied zur Universität stellen NONSTANDARD-Varietäten an der Schule für manche Lehrpersonen auch eine Möglichkeit dar, ein Bewusstsein für eine 
situationsangemessene Varietätenverwendung bei den Lernenden $\mathrm{zu}$ schaffen. LC4 zufolge ist es wichtig, die „sprachlichen Register für formelle und informelle Kontexte“ bedienen zu können. Das schließe auch den DiALEKT mit ein. Die Lernenden sollten auf verschiedene sprachliche Anforderungen vorbereitet werden und dafür sensibilisiert werden, sich in verschiedenen sozialen Kontexten sprachlich angemessen zu verhalten. Nach Ansicht von LC4 trägt genau diese sprachliche Diversität dazu bei, dass man für andere Varietäten und Sprachen sensibilisiert und im Zuge dessen „toleranter“ anderen Sprachformen gegenüber werde (15:41-16:12). Zudem würde die Auseinandersetzung mit anderen Varietäten auch zu einer Veränderung des persönlichen Sprachgebrauchs und in weiterer Folge zu einem Ausbau STANDARDSPRACHLICHER Kompetenzen beigetragen. Das sei die Voraussetzung für eine umfassende Sprachkompetenz. Die Schule sollte als „Übungsplatz“ dafür dienen.

Generell wird an der Schule - im Unterschied zur Universität - davon ausgegangen, dass Kompetenzen im STANDARD bei den Lernenden nicht von Anfang an gegeben sind. Auch wenn STANDARD für LG4 in der Schule eine „wichtige Varietät“ sei, die auch die „größte kommunikative Reichweite“ aufweise, sei er für viele Schüler/innen de facto aber „mündlich nur schwer realisierbar“. Aus diesem Grund würden STANDARDABWEICHENDE Sprechweisen akzeptiert, um die Angst vor Überforderung angesichts zu hoher STANDARD-Erwartungen zu minimieren. So finde die Alltagskommunikation der meisten Schüler/innen im ländlichen Raum ausschließlich im DIALEKT statt (15:20-16-11). LC2 zufolge sei der „Wechsel“ zum STANDARD „sehr schwierig“. Der DiALEKT sei somit eine Art „Hilfsvarietät“, die „natürlich mit Grenzen“ akzeptiert werde, um den Schüler/inne/n entgegenzukommen (06:15-07:22).

Stellt der NONSTANDARD vorwiegend für Deutschlehrer/innen eine Hilfestellung dar, wird er in anderen Fächern großteils als die eigentliche Unterrichtssprache definiert. So fällt es LC1 zufolge in anderen Gegenständen „nur zum Teil“ in den Aufgabenbereich einer Lehrperson, auf die Sprache zu achten. Solange eine gegenseitige Verständigung gewährleistet sei, sei der DIALEKT dort durchaus adäquat (05:21-06:15). Auch SA1 argumentiert dahingehend, dass die Sprache in anderen Fächern lediglich „als Medium“ diene, explizit aber „kaum etwas mit dem Lernstoff zu tun“ habe. Insofern könne man durchaus gute Leistungen beispielsweise in Mathematik erbringen, ohne STANDARDSPRACHE im Unterricht zu sprechen (07:39-08:25). Ähnlich pragmatisch wird auch bisweilen an der Universität argumentiert (vgl. Abschnitt 4.1.2). 


\section{Diskussion und Fazit}

Im vorliegenden Beitrag präsentierten wir Ergebnisse einer qualitativen Untersuchung zu Varietätenkonzeptionen an Schulen in Salzburg und Tirol und an der Universität Salzburg. In Anlehnung an neuere qualitative Ansätze der Perzeptionslinguistik wurden für den vorliegenden Beitrag keine quantitativen Daten, ${ }^{11}$ sondern ausschließlich Gesprächsdaten ausgewertet, die im Rahmen halbstrukturierter Interviews mit insgesamt 264 GP erhoben wurden. Begründen lässt sich diese Vorgehensweise damit, dass die inhaltsanalytische Auswertung von Interviewdaten eine Tiefe der Rekonstruktion abstrakter Konzepte wie den Varietätenkonzeptionen gestattet, die rein quantitative Ansätze nicht zu leisten vermögen. Die Interviewdaten wurden daraufhin ausgewertet, (1) welche Varietätenkonzeptionen sich aus den Äußerungen erschließen lassen, (2) wie die Verwendung dieser Varietäten innerhalb und außerhalb des Schul- bzw. Universitätsalltags bewertet wird sowie (3) wann welche Varietäten aus der Sicht der GP verwendet werden sollten (und wann nicht).

Generell lässt sich festhalten, dass zwischen den Varietätenkonzeptionen der GP an der Universität und den Schulen viele Gemeinsamkeiten auftreten. Was beiden Institutionstypen gemeinsam ist, sind die heterogenen Sprachverhältnisse in Bezug auf innere wie äußere Mehrsprachigkeit, wobei die Heterogenität an der Universität aufgrund des höheren Anteils von Personen, die nicht in Österreich bzw. überhaupt im deutschsprachigen Raum aufgewachsen sind, ausgeprägter ist als an den Schulen (vgl. Abschnitt 3). Mit Blick auf die drei genannten Fragen lässt sich aus den Analysen Folgendes rekonstruieren:

1. Alle GP haben ein klares Bewusstsein für die Existenz von mindestens zwei Varietäten. Das STANDARDDEUTSCHE wird meist als HOCHDEUTSCH bezeichnet, der NONSTANDARD meist als DIALEKT, gelegentlich wird zusätzlich auch von einer „UMGANGSSPRACHE“ oder einfach von einem „DAZWISCHEN“ (von Lehrer/inne/n an den Schulen) gesprochen. Bei jenen GP, deren Varietätenkonzeption neben HochDEUTSCH und DIALEKT keine(n) Zwischenbereich(e) vorsieht, muss man annehmen, dass der DiALEKT-Begriff sehr weit ist und neben den Basisdialekten auch den mittleren Bereich umfasst.

Von GP an Schulen wie an der Universität werden DIALEKTE hauptsächlich als informelle, nähesprachliche Varietäten begriffen; der STANDARD/das HocHDEUTSCHE wird entsprechend als formelle, distanzsprachliche Varietät

$11 \mathrm{Zu}$ ausgewählten quantitativen (neben qualitativen) Ergebnissen vgl. Vergeiner et al. (2019). 
konzeptualisiert. Auffällig ist an beiden Institutionen, dass STANDARDSPRACHLICHKEIT dabei stark mit Schriftlichkeit assoziiert wird. Insbesondere an den Universitäten lassen viele GP ein starres, monozentrisches, auf die Existenz einer homogenen Entität gerichtetes Verständnis von STANDARD erkennen, einige andere eher ein an einen GEBRAUCHSSTANDARD erinnerndes Konzept, das etwa auch österreichische Eigenheiten zulässt. Die Vorstellung eines eigenen ÖSTERREICHISCHEN HOCHDEUTSCH klingt nur in einigen Interviews mit Schüler/inne/n an - allerdings meist mit dem Hinweis einer gewissen Höherwertigkeit des DEUTSCHEN HochDEUTSCH. Wenn es in den Köpfen dieser GP also tatsächlich so etwas wie ein Konzept der Plurizentrizität gibt, dann ist es klar von einer Asymmetrie zugunsten des BUNDESDEUTSCHEN STANDARDS geprägt - so, wie es etwa für das Verhältnis zwischen DEUTSCHSCHWEIZERISCHEM und BUNDESDEUTSCHEM STANDARD beschrieben wird (vgl. Scharloth 2005).

2. Die Bewertungen der Varietätenverwendungen lassen sich zum Teil expliziten Urteilen entnehmen, zum Teil müssen sie aus Äußerungen, die ihnen bestimmte Attribute zuschreiben, erschlossen werden. Explizit sind etwa den DiALEKT stigmatisierende Bewertungen, die an Vorstellungen von einem RESTRINGIERTEN CODE erinnern. Gemeinsam ist Schule und Universität dabei etwa, dass der NONSTANDARD vielfach als falsche, inkorrekte Sprechweise attribuiert wird. $\mathrm{Zu}$ den indirekten Bewertungen gehören die an beiden Institutionstypen anzutreffenden Äußerungen, wonach dem STANDARD im Vergleich zu NONSTANDARD-Varietäten ein hoher Grad an Verständlichkeit attestiert wird - an der Universität nicht nur mit Blick auf Nichtmuttersprachler/innen des Deutschen, sondern durchaus auch auf bundesdeutsche Studierende und Lehrende (vgl. ausführlicher dazu Vergeiner 2020). Aus der These der besseren Verständlichkeit des STANDARDs lässt sich die Auffassung ableiten, dass sich für bestimmte universitäre Verwendungssituationen, z. B. Lehrveranstaltungen, per se nur STANDARD-Varietäten eigneten (siehe Punkt 3).

3. Was den schulischen Bereich betrifft, zeigen sich bei den indirekten Bewertungen von Varietätenverwendungen klare Unterschiede zwischen Schüler/inne/n, die städtische und solchen, die ländliche Schulen besuchen. Während Schüler/innen an Schulen im städtischen Umfeld den STANDARD als „Sprache des täglichen Umgangs“ charakterisieren, kommt dieses Attribut am Land den DiALEKTEN zu. Schüler/innen im ländlichen Umfeld etikettieren das HocHDEUTSCHE vielfach als so etwas wie ihre erste Fremdsprache, als Sprache der Gebildeten bzw. der Bildung oder als Sprache der Schrift auf jeden Fall als eine exogene Varietät. Auch hier bestehen durchaus $\mathrm{Pa}$ - 
rallelen zu Sprachkonzeptionen von Deutschschweizer/inne/n (vgl. Christen \& Schmidlin 2019).

4. Aus verschiedenen Attribuierungen und Bewertungen ergeben sich schließlich auch Erwartungen an Varietätenverwendungen. Wenn etwa auf der einen Seite Universitätslehrer/innen den Gebrauch des STANDARDS mit Kompetenz und Professionalität oder Studierende mit wissenschaftlicher Seriosität assoziieren, so ergibt sich daraus fast zwangsläufig, dass solche Akteure und Akteurinnen für universitäre Lehrveranstaltungen und Prüfungsgespräche die Verwendung der STANDARDSPRACHE einfordern - wie auch immer sich diese am Ende wirklich anhört. Wird auf der anderen Seite - von Universitätsangehörigen wie auch von Lehrenden und Schüler/inne/n an den Schulen gleichermaßen - hervorgehoben, dass regionale NONSTANDARD-Varietäten wichtige identitätsstiftende Funktionen hätten und die Sprecher/innen ,authentischer' wirken ließen, so wird damit ihre Verwendung in Unterrichtssituationen legitimiert. Eine ganz eigene Rechtfertigung des NONSTANDARD-Gebrauchs im Unterricht findet sich in Äußerungen von Lehrer/inne/n vor allem ländlicher Schulen: Da DIALEKTE so stark in der Alltagskommunikation vieler Schüler/innen verankert und auch durchaus positiv konnotiert seien, sei die Verwendung von NonSTANDARD-Varietäten im Unterricht nicht nur üblich, sondern in einem bestimmten Rahmen auch angemessen und legitim. Wichtig sei mit Blick auf mündliche Abschlussprüfungen und das spätere Berufsleben eine Sensibilisierung für situationsangemessene Varietätenverwendung, die eben den Gebrauch der STANDARD- wie der NONSTANDARD-Varietäten im Unterricht umfasse.

In inhaltlicher Perspektive fördern die Ergebnisse unserer Untersuchungen sowohl heterogene als auch sehr reflektierte Varietätenkonzeptionen von Akteur/inn/en im sekundären und im tertiären Bildungsbereich in Österreich zutage. Dabei dürften sie, wie überhaupt die Salzburger Sprachverhältnisse aufgrund ähnlich diaglossischer Situationen -, mit denen in anderen Regionen in Österreich sowie im Süden Deutschlands vergleichbar sein. Wie gezeigt, besteht auch eine gewisse Vergleichbarkeit der Varietätenkonzeptionen mit denen in der Schweiz, trotz der grundlegend anderen, Situation dort. Für die Verhältnisse an mittel- und norddeutschen Schulen und Universitäten, die weitgehend vom monovarietären Gebrauch von STANDARDNAHEN Varietäten - oder zumindest einem monovarietären Habitus - geprägt sind, mögen sich die drei genannten Untersuchungsfragen hingegen erst gar nicht stellen. 
In methodischer Hinsicht zeigt sich der Wert qualitativer Ansätze, die zum einen eine analytische Tiefe erreichen, welche bei rein quantitativen Zugängen nicht möglich wäre, und die zum anderen durchaus eine Generalisierbarkeit der Ergebnisse beanspruchen können, wenn sie sich - wie im vorliegenden Fall auf eine große Zahl von GP stützen. Dadurch wird natürlich der Wert quantitativer Untersuchungen keineswegs in Abrede gestellt; vielmehr können sich der quantitative und der qualitative Ansatz gegenseitig sinnvoll ergänzen - wie in den beiden Projekten, in deren Rahmen der vorliegende Beitrag entstanden ist.

\section{Literaturverzeichnis}

Ajzen, Icek (1989): Attitude structure and behavior. In Anthony R. Pratkanis, Steven J. Breckler \& Anthony G. Greenwald (Hrsg.), Attitude structure and function, 241-274. Hillsdale: Lawrence Erlbaum.

Allport, Gordon W. (1935): Attitudes. In Carl Murchison (Hrsg.), A handbook of social psychology. Worcester: Clark University Press.

Ammon, Ulrich (1972): Dialekt, soziale Ungleichheit und Schule. Weinheim: Beltz.

Anders, Christina Ada (2010): Wahrnehmungsdialektologie. Das Obersächsische im Alltagsverständnis von Laien. Berlin: De Gruyter.

Bartsch, Renate (1987): Sprachnormen. Theorie und Praxis. Tübingen: Niemeyer.

Bellamy, John (2012): Language Attitudes in England and Austria. A Sociolinguistic Investigation into Perceptions of High and Low-Prestige Varieties in Manchester and Vienna. Stuttgart: Steiner.

Berger, Peter L. \& Thomas Luckmann (2016): Die gesellschaftliche Konstruktion der Wirklichkeit. Eine Theorie der Wissenssoziologie. 26. Aufl. Frankfurt a. M.: Fischer.

Beuge, Patrick (2019): Was ist gutes Deutsch? Eine qualitative Analyse laienlinguistischen Sprachnormwissens. Berlin, Boston: De Gruyter.

Cavanaugh, Jillian R. (2013): Language ideologies and language attitudes. A linguistic anthropological perspective. In Peter Auer, Javier Caro Caro Reina \& Göz Kaufmann (Hrsg.), Language variation - European perspectives IV. Selected papers from the Sixth International Conference on Language Variation in Europe (ICLaVE 6), Freiburg, June 2011, 45-55. Amsterdam: Benjamins.

Christen, Helen \& Regula Schmidlin (2019): Die Schweiz. Dialektvielfalt in mehrsprachigem Umfeld. In Rahel Beyer \& Albrecht Plewnia (Hrsg.), Handbuch des Deutschen in West- und Mitteleuropa: Sprachminderheiten und Mehrsprachigkeitskonstellationen, 193-244. Tübingen: Narr Francke Attempto.

Cillia, Rudolf de (2018): Sprache(n) im Klassenzimmer. Varietätengebrauch und Spracheinstellungen in der Schule aus Sicht der Lehrer/innen und der Schüler/innen. In Monika Dannerer \& Peter Mauser (Hrsg.), Formen der Mehrsprachigkeit in sekundären und tertiären Bildungskontexten. Verwendung, Rolle und Wahrnehmung von Sprachen und Varietäten. Unter Mitarbeit von Philip C. Vergeiner, 67-85. Tübingen: Stauffenburg.

Coupland, Nikolas \& Adam Jaworski (2004): Sociolinguistic perspectives on metalanguage: Reflexivity, evaluation and ideology. In Adam Jaworski, Dariusz Galasiński \& Nikolas Cou- 
pland (Hrsg.), Metalanguage. Social and ideological perspectives, 15-51. Berlin, New York: De Gruyter.

Cuonz, Christina (2014): Was kann die diskursive Spracheinstellungs forschung (nicht)? Methodologische und epistemologische Überlegungen. In Christina Cuonz \& Rebekka Studler (Hrsg.), Sprechen über Sprache. Perspektiven und neue Methoden der Spracheinstellungsforschung, 31-64. Tübingen: Stauffenburg.

Dannerer, Monika (2018): Sprachwahl, Sprachvariation und Sprachbewertung an der Universität. In Arnulf Deppermann \& Silke Reineke (Hrsg.), Sprache im kommunikativen, interaktiven und kulturellen Kontext, 169-192. Berlin, Boston: De Gruyter.

Elspaß, Stephan \& Konstantin Niehaus (2014): The standardization of a modern pluriareal language. Concepts and corpus designs for German and beyond. Orð og tunga 16, 47-67.

Elspaß, Stephan \& Stefan Kleiner (2019): Forschungsergebnisse zur arealen Variation im Standarddeutschen. In Joachim Herrgen \& Jürgen Erich Schmidt (Hrsg.), Sprache und Raum Ein internationales Handbuch der Sprachvariation. Band 4: Deutsch, 159-184. Berlin, Boston: De Gruyter.

Errington, Joseph (1999): Ideology. Journal of Linguistic Anthropology 9 (1), 115-117.

Garrett, Peter, Nikolas Coupland \& Angie Williams (2003): Investigating language attitudes: Social meanings of dialect, ethnicity and performance. Cardiff: University of Wales Press.

Gloy, Klaus (1975): Sprachnormen I. Linguistische und soziologische Analysen. Stuttgart: Frommann-Holzboog.

Gloy, Klaus (2004): Norm. In Ulrich Ammon, Norbert Dittmar, Klaus J. Mattheier \& Peter Trudgill (Hrsg.), Sociolinguistics. An international handbook of the science of language and society. Volume 1. 2nd completely rev. and extended ed, 392-399. Berlin, New York: De Gruyter.

Hochholzer, Rupert (2004): Konfliktfeld Dialekt. Das Verhältnis von Deutschlehrerinnen und Deutschlehrern zu Sprache und ihren regionalen Varietäten. Regensburg: Edition Vulpes.

Hundt, Markus (2009): Normverletzungen und neue Normen. In Marek Konopka \& Bruno Strecker (Hrsg.), Deutsche Grammatik - Regeln, Normen, Sprachgebrauch, 117-140. Berlin: De Gruyter.

Hundt, Markus, Nicole Palliwoda \& Saskia Schröder (2015): Der deutsche Sprachraum aus der Sicht linguistischer Laien - das Kieler DFG-Projekt. In Michael Elmentaler, Markus Hundt \& Erich Jürgen Schmidt (Hrsg.), Deutsche Dialekte. Konzepte, Probleme, Handlungsfelder. Akten des 4. Kongresses der Internationalen Gesellschaft für Dialektologie des Deutschen (IGDD) in Kiel, 295-322. Stuttgart: Steiner.

Irvine, Judith. T. \& Susan Gal (2000): Language Ideology and Linguistic Differentiation. In Paul V. Kroskrity (Hrsg.), Regimes of language. Ideologies, polities, and identities. 1. Aufl., 3584. Santa Fe, NM: School of American Research Press.

Knöbl, Ralf (2012): Dialekt - Standard - Variation. Formen und Funktionen von Sprachvariation in einer mittelschwäbischen Schulklasse. Heidelberg: Winter.

Krefeld, Thomas \& Elissa Pustka (2010): Für eine perzeptive Varietätenlinguistik. In Thomas Krefeld \& Elissa Pustka (Hrsg.), Perzeptive Varietätenlinguistik, 9-28. Frankfurt a. M.: Lang.

Kroskrity, Paul V. (2009): Language Ideologies. In Alessandro Duranti (Hrsg.), A Companion to Linguistic Anthropology. 4. Aufl., 496-517. Malden: Blackwell.

Lambert, Wallace E., Richard Hodgson, Robert C. Gardner \& Samuel Fillenbaum (1960): Evaluational reactions to spoken languages. Journal of Abnormal and Social Psychology 60 (1), 44-51. 
Liebscher, Grit \& Jennifer Dailey-0'Cain (2009): Language attitudes in interaction. Journal of Sociolinguistics 13 (2), 195-222.

Lasagabaster, David (2004): Attitude / Einstellung. In Ulrich Ammon, Norbert Dittmar, Klaus J. Mattheier \& Peter Trudgill (Hrsg.), Sociolinguistics. An international handbook of the science of language and society. Volume 1. 2nd completely rev. and extended ed., 399-405. Berlin, New York: De Gruyter.

Mattheier, Klaus J. (1991): Standardsprache als Sozialsymbol. Über kommunikative Folgen gesellschaftlichen Wandels. In Rainer Wimmer (Hrsg.), Das 19. Jahrhundert. Sprachgeschichtliche Wurzeln des heutigen Deutsch, 41-72. Berlin, New York: De Gruyter.

Moosmüller, Sylvia (1991): Hochsprache und Dialekt in Österreich. Soziophonologische Untersuchungen zu ihrer Abgrenzung in Wien, Graz, Salzburg und Innsbruck. Wien: Böhlau.

Preston, Dennis R. (2009): Language, space and the folk. In Peter Auer \& Jürgen Erich Schmidt (Hrsg.), Language and Space. An International Handbook of Linguistic Variation. Volume 1: Theories and Methods, 179-201. Berlin: De Gruyter.

Purschke, Christoph \& Philipp Stoeckle (2019): Perzeptionslinguistik arealer Sprachvariation im Deutschen. In Joachim Herrgen \& Jürgen Schmidt (Hrsg.), Sprache und Raum Deutsch. Ein internationales Handbuch der Sprachvariation, 844-860. Berlin: De Gruyter.

Scharloth, Joachim (2005): Asymmetrische Plurizentrizität und Sprachbewusstsein. Einstellungen der Deutschschweizer zum Standarddeutschen. Zeitschrift für Germanistische Linguistik 33 (2), 236-267.

Schoel, Christiane \& Dagmar Stahlberg (2012): Spracheinstellungen aus sozialpsychologischer Perspektive II: Dialekte. In Ludwig M. Eichinger, Albrecht Plewnia, Christiane Schoel \& Dagmar Stahlberg (Hrsg.), Sprache und Einstellungen. Spracheinstellungen aus sprachwissenschaftlicher und sozialpsychologischer Perspektive. Mit einer Sprachstandserhebung zum Deutschen von Gerhard Stickel. 1. Aufl, 205-225. Tübingen: Narr Francke Attempto.

Silverstein, Michael (1992): The uses and utility of ideology: Some reflections. Special Issue of Pragmatics 2 (3), 311-323.

Soukup, Barbara (2009): Dialect use as interaction strategy. A sociolinguistic study of contextualization, speech perception, and language attitudes in Austria. Wien: Braumüller.

Steiner, Astrid (2008): Unterrichtskommunikation. Eine linguistische Untersuchung der Gesprächsorganisation und des Dialektgebrauchs in Gymnasien der Deutschschweiz. Tübingen: Narr.

Vergeiner, Philip C. (2019): Kookkurrenz - Kovariation - Kontrast. Formen und Funktionen individueller Dialekt-/Standardvariation in Beratungsgesprächen. Wien: Peter Lang.

Vergeiner, Philip C. (2020): Sprachnormbezogene Akkommodation in der Hochschullehre. Zeitschrift für Angewandte Linguistik 72, 33-62. DOI: https://doi.org/10.1515/zfal-20202027 (letzter Zugriff 02.03.2020).

Vergeiner, Philip C. (2021): Bewertungen - Erwartungen - Gebrauch: Sprachgebrauchsnormen zur inneren Mehrsprachigkeit an der Universität. Stuttgart: Steiner.

Vergeiner, Philip C., Elisabeth Buchner, Eva Fuchs \& Stephan Elspaß (2019): Sprachnormvorstellungen in sekundären und tertiären Bildungseinrichtungen in Österreich. Zeitschrift für Dialektologie und Linguistik 86 (3), 284-330.

Woolard, Kathrvn A. (1992): Language Ideology: Issues and Approaches. Pragmatics 2 (3), 235249.

Woolard, Kathrvn A. \& Bambi B. Schieffelin (1994): Language Ideology. Annual Review of Anthropology 23, 55-82. 